\title{
Hypothesis of Cancer Stem Cells and Stem Cells of Head and Neck Cancer-Biomarkers for their Identification
}

\author{
Mladen Vukovic* \\ Department of Dentistry, Serbia \\ *Corresponding author: Mladen Vukovic, DMD, PhD student at the Faculty of Dentistry, Serbia
}

Submission: 海 October 05, 2018; Published: 監 November 26, 2018

\begin{abstract}
Stem cells are characterized by their self-renewal capacity and the ability to differentiate into any type of mature cell. In addition to normal stem cells, cancerous stem cells have been found to be responsible for the growth and spread of tumors. According to the CSC hypothesis CSC leads to tumorigenesis and tumor growth. Head and neck cancer are the sixth most common cancer and about 300,000 new cases are diagnosed every year. Despite progress in treatment, the 5-year survival rate for this cancer has not improved over the past 30 years. In the hierarchical structure of stem cells present in the human oral epithelium, it is clear that stem cells are the only long-lived inhabitants of the oral epithelium and, therefore, the only cells capable of accumulating the required number of genetic changes for the development of malignancy. The major surface phenotypic markers associated with SC and CSC characteristics include CD133, CD44 and CD24.
\end{abstract}

Abbreviations: CSC: Cancer Stem Cells; SC: Stem Cells; HNSCC: Head and Neck Squamous Carcinoma Cells; ATC: Amplifying Transitory Cells; NOD/ SCID: Non-obese diabetic/Severe Combined Immunodeficiency; OSCC: Oral Squamous Cell Carcinoma; DNA: Deoxyribonucleic Acid; EMT: Epithelial Mesenchymal Transition; SP: Sidepopulation; HPV: Human Papillomavirus; HNC: Head and Neck Cancer; UADT: Upper Aerodigestion Cancer

\section{Introduction}

This paper deals with the study of the head and neck carcinoma stem cell hypothesis, the various factors of identification and their impact on tumor progression, their clinical and therapeutic implications. Also, I analyze cancer association and its relationship with stem cell carcinogenesis. Recovery of normal tissue depends on subpopulation of cells known as the stem cells characterized by extensive proliferative potential. Stem cells are distributed in vivo with respect to epithelial cell units and epithelial stem cells are usually divided, reconstructed and produced by transitive amplification cells that pass through a series of divisions with simultaneous differentiation. Malignant neoplasms are believed to result from sequential mutations resulting from progressive genetic instability and/or impact of environmental factors. Reya et al. [1] have supported the hypothesis that cancer can develop due to mutations in stem cell populations because of the fact that they are the only cells permanently inhabited in the tissue, they are constantly exposed to the carcinogenic effects and, therefore, the development of epithelial tumors involves the initial genetic changes in the stem cells themselves [1].

According to the CSC theory only specific subpopulation of cancer stem cells has the ability to maintain cancer growth. The four key characteristics define the CSC population: (a) only a small proportion of cancer cells within the tumor have tumorigenic potential when transplanted into immunodeficiency mice; (b) The sub-population of the CSC can be separated from other cancer cells by recognizable cell surface markers; (c) a tumor originating from a CSC comprises a mixture of tumorigenic and non-tumorigenic cells of the original tumor; and (d) CSC subpopulation can be serially transplanted over several generations indicating the self-renewing potential of the population.

To date, human CSCs have been identified and isolated in various types of malignant tumors, including breast, brain, prostate, ovary, head and neck cancer; Moreover, several studies have shown that treatment with drugs or radiation can strengthen and stimulate the maintenance of CSC subpopulation in vitro and in vivo, pointing to CSC's responsibility for tumor regeneration. Today, mainly for proving CSC in different organs, xenograft tests are used. In accordance with the 2006 AACR suggestions of the CSC, an orthotopic xenograft test is considered to be the gold standard for CSC identification [1].

\section{Stem Cells}

Stem cells are characterized by their capacity for self-renewal and the ability to differentiate into any type of mature cell [1]. Embryonic stem cells can be differentiated into three cell lines from which all types of tissue can be formed (Martin 1981; Evans and Kaufman 1981) while stem cells are excluded from an adult organism immature cells that can only be differentiated into cell 
types present in the host tissues which can be updated. Their capacity for self-renewal allows SC to survive throughout the life of individual. They must be able to restore and maintain a balance between self-renewal and differentiation and the preservation of tissue homeostasis. If cancer is a truly regulatory disorder of the SC to self-regenerate, it is important to determine molecular mechanisms that regulate normal SC self-replication to understand the processes that lie in the basis of tumor cell proliferation [1].

\section{Cancer Stem Cells (CSC)}

It is currently considered that a small group of tumor cells known as CSCs have the ability to self-restore and produce phenocopy of the original tumor [2], which is derived from the bulk of tumor cells and which coordinate tumor growth. The idea that the CSC are responsible for tumorigenesis is derived from heterogeneous tumor composition including OSCC. Histological studies reveal different tumor regions with varying degrees of differentiation; while genetic studies show a similar heterogeneity to areas that differ in the expression of the gene and hence in the expression of the protein; the cells in the tumor differ in the rate of proliferation and the ability to generate new tumors. As mentioned above, it has been suggested that a small CSC cell population with a recognizable phenotype is responsible for the growth of a new tumor $[1,3,4]$. Their existence has been considered for similarities in the histological profile and the pattern of proliferation between well-differentiated tumors and the epithelium of their origin.

CSCs are described as a SC type that differentiates in aberrant form and can produce tumor cell populations that are phenotypically different. According to the CSC hypothesis, CSC leads to tumorigenesis and tumor growth. However, the knowledge of CSC and normal SC in healthy or tumor tissue makes more difficult the lack of specific SC and CSC markers especially in the oral and squamous cell and head squamous cell carcinoma (HNSCCs) [5]). It has recently been reported that HNSCC contains less than one CSC per 2500 cells [6]). CSCs are often organized in three sections, as in the normal epithelium (basal CSC, ATC, and the furthest differentiated cell section). This similarity in the proliferative hierarchy with the non-tumor oral epithelium suggests that tumor growth is maintained by one type of tumor cell CSC (Figure 1); [7].
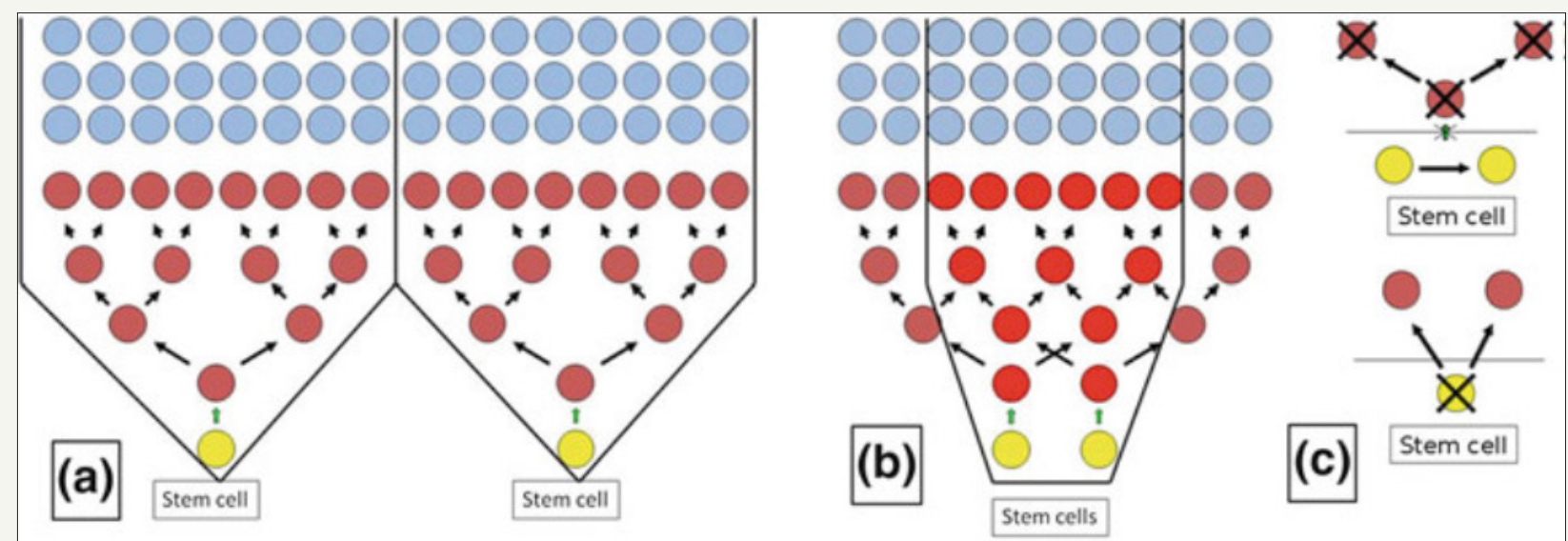

Figure 1: (a) In each division, normal SC (yellow circles) generate identical SC and ATC. ATC (brown circles) are divided 3-5 times until the differentiation of all daughter cells ends. This asymmetric hierarchical proliferation envisages a theoretical spatial arrangement of the offspring in the form of inverted pyramidal structures (proliferative units-within black lines). (b) Areas of intersections (red circles) with adjacent proliferative units, in which epithelial regeneration depends on coordinated and variable activity of different SCs. (c) above: a symmetric division of the SC resulting in two SCs; below: symmetric SC division, resulting in two ATCs with loss of SC.

Bonnet and Dick (1997) found that a small group of tumor cells with CD34+/CD38 phenotype in acute myeloid leukemia in humans (AML) were able to generate AML when transplanted to NOD/SCID mice but other tumor cells were not. They suggested that human AML was organized as a hierarchy of cells derived from primitive hematopoietic cells. Similarly, tumors of breast cancer have been generated in NOD/SCID mice with a small group of CD44+/CD24 cells, but not by 100 times the number of CD44 cells. CSCs have been proven in other solid tumors [2-8] including HNSCC and OSCC in which there was only a small subset of CD44+ cells capable of generating a tumor [3].

\section{Stochastic Model of Tumor Origin and CSC Hypothesis}

According to the stochastic model malignant transformation is a consequence of random mutations of any cell thus creating a tumor through clonal progression of mutants-progeny cells that possess proliferative ability and produce genomic instability, leading to the accumulation of epigenetic/genetic events and the selection of progressively more aggressive subclones [9-11]. The development of these subclones of different phenotypes and proliferative capacities is responsible for tumor heterogeneity. In this model cancer is considered as a proliferative disease [8].

The CSC hypothesis has two components. The first component is associated with cellular tumor origin and increased risk of accumulation of multiple mutations over a long life of SC (Figure2); [8]. It has been estimated that malignant transformation of the normal cells requires 3-6 oncogenic events. A genetic model of progression in OSCC has been proposed linking the genetic changes with phenotypic progression to malignancy and indicating that the accumulation of sufficient mutations for the development of OSCC would be possible only in long-lived cells. In addition, SC and 
CSC have the capacity for self-renewal and self-renewal disorder is an early and crucial event in carcinogenesis. The self-renewal mechanism is activated in normal SC and maintained more active than de-activation in more differentiated cells. It is suggested that the CSC have the appropriate self-restraining mechanism similar to the normal SC. Animal studies implied disregulation of molecular pathways involved in self-regeneration (Vnt, Notch and Hedgegog) in tumorigenesis [8].

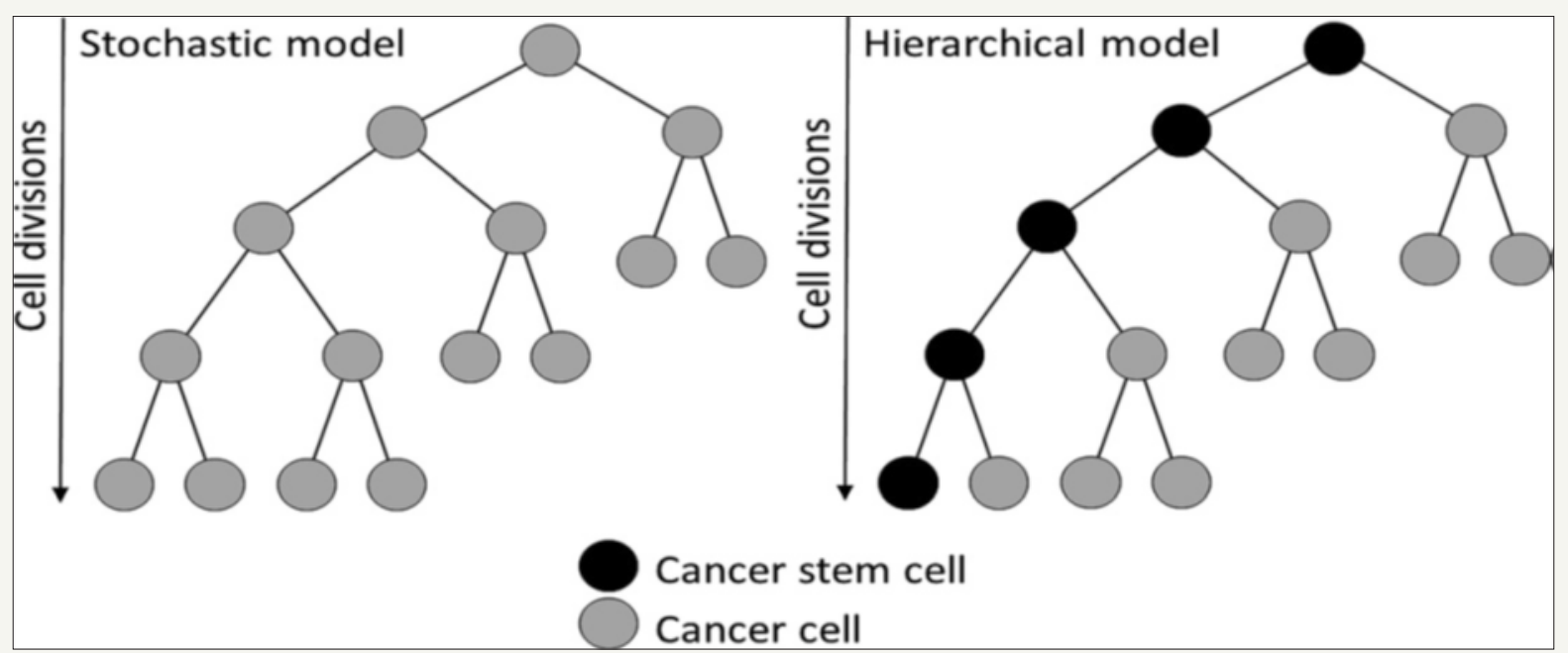

Figure 2: In the stochastic model, all tumor cells have the same abilities to propagate, initiate tumors and wider metastases. The tumor heterogeneity in this model was derived from spontaneous phenotypic changes. The proposed CSC hypothesis dictates a hierarchical model in which an asymmetric division results in specific and well-defined populations of cancer stem cells and other cancer cells that do not initiate tumors or wider metastases. These cells may consist of populations with reduced proliferative capacity (i.e. cells that enhance transit) or post-mitotic differentiated cells without further proliferative ability or activity.

The second component of the CSC hypothesis is that the CSC is responsible for maintaining tumor growth, which is supported by the findings that SC transplantation into NOD/SCID mice not only enhanced self-reproducibility of the cells but also promoted tumor growth through their uneven differentiation capacity, reproducing the heterogeneity of the original tumor and Dick 2005.

If the CSC hypothesis is correct, normal SCs must have a powerful anti-cancer mechanism to reduce the risk of malignant transformation. One mechanism could be their asymmetric pattern of division which leads to ATC's proliferation, differentiation, and desquamation and the prevention of the accumulation of mutations in ATC that suffer from initial oncogenic events. The SC divide rate is also low in asymmetric divisions reducing the risk of mutation in any mitosis and may be even lower if the recovery area is affected by more than one SC. Another anti-cancer mechanism may include selective segregation of DNA in the asymmetric division of the SC as observed in SC in the intestine and breast with the secretion of newly synthesized DNA from SC to ATC. In fact, the SC adult gene must be highly stable because of the DNA repair mechanisms during the life of an individual must be maintained and preserved. Thus, a high incidence of cancer is observed in syndromes with defects in repair of the DNA (eg, telangiectasia and keroderma pigmentosum ataxia). Some results indicate that CSCs can undergo changed behaviors including resistance to therapy as a result of chromosomal instability due to defects in chromosome segregation. However, the creation of SCs requires the reprogramming of differentiated cells, the maintenance of which is essential; Therefore, this reprogramming would require the inclusion of powerful transcription controllers and/or epigenetic machinery. Four transcription factors participate in reprogramming in oncogenesis and early inhibition of somatic gene involved in differentiation by c-Mic, which is crucial. Histone deacetylase inhibitors can replace c-Mic in reprogramming, participating in the suppression of differentiated cells - the program of gene expression. Transcription factors OCT-4, Sok-2, Klf-4 and 4ITF are involved in reprogramming at a later stage [8].

It has recently been reported that different cancer cells can become CSCs through an epithelial mesenchymal transition (EMT), in which epithelial cells acquire mesenchymal characteristics under the influence of specific environmental stimuli and can expand into surrounding tissues and distant organs. EMT is promoted by the activity of transcription factors such as Snail, Tvist 1 and ZEB 1 that change polarity epithelial cells and suppress the expression of E-cadherin, among others [8].

\section{Identification of SC and CSC}

To facilitate the identification and purification of normal SC and CSC, some specific markers on the cell surface were investigated and several markers of normal stem cells were common for CSC in different types of tumors. The standard procedures for isolating CSC are similar in many studies. Among the most commonly used in vivo models is the fractionation of tumor cells using cell surface markers with stem cell characteristics followed by their implantation in NODSCID mice to allow xenograft growth. The major surface phenotypic markers associated with stem cell characteristics include CD133, CD44 and CD24.

CD133, also called prominin 1 (PROM1), is a glycoprotein of the cell surface, with five transmembrane domains and two larges glycosylate extra-cell rings. It was discovered as a marker of normal hematopoietic stem cells and was later used to purify CSC in several types of tumors. His power to identify the cell with the stem cell 
characteristics was confirmed in recent studies. Intracoronary infusion of CD133+ endothelial progenitor cells improves cardiac function and quality of life of patients with chronic postinfarct heart failure. The CD133 + cancer cell shows the same possibilities. High levels of CD133 expression have been accepted as an important marker for several cell lines of cancer. That is why Clarke et al. [2] defines prominin as a "momentum molecule". The presence of CD133 was detected in several solid tumors of various anatomical sites, such as: central nervous system cancer, prostate cancer, pancreatic cancer, colorectal cancer, liver cancer, melanoma, breast cancer, lung cancer, laryngeal cancer and osteosarcoma. In conclusion, the CD133 is able to identify and isolate the CSC [3].

CD44 is a cellular surface glycoprotein receptor for hyaluronic acid and appears to be involved in cell adhesion, cell migration and metastasis of cancer cells. Hyaluronic acid (HA) is one of the main components of the extracellular matrix and the most important basic substance of connective tissue. It shows important biological features and plays an important role in key physiological processes, especially in inflammatory processes, immunological reactions, angiogenesis and wound healing.

It is also strongly involved in the migration of neoplastic cells and therefore in the metastatic spread of malignant tumors. The CD44 marker of the cell surface was used to identify CSC in many types of tumors, such as tumors of the breast, prostate, pancreas, and head and neck cancer. It is one gene of $60 \mathrm{~kb}$ heavy, containing 20 exons mapped to the locus $11 \mathrm{p} 13$ chromosome. The different isoforms of the CD44 variant are differently expressed in normal and malignant cells. CD44 is a surface glycoprotein that acts as an intercellular receptor or a matrix receptor as the carrier of the signal and growth factor. Several human tumors show changed expression and alteration of CD44, with further change in CD44 during tumor progression. In lung cancer, CD44v5 and v6 are dominant forms that promote metastases in squamous cell carcinoma and bronchoalveolar carcinoma; in colon cancer, CD44v3 seems to promote invasion and resistance to apoptosis, while CD44v6 is associated with metastases and a reduction in survival rates; in breast cancer CD44v3 promotes metastases and progression of cancer. These observations indicate an important role for CD44 in tumor progression, cell transformation, tumor diagnosis, and prognosis. In conclusion, the CD44 could have several functions related to the increase, progression and metastatic cancer. It is not only a hyaluronic acid receptor, but it is also capable of cooperating with many matrix proteins such as fibronectin, type I and type IV collages, serglicin and osteopontin, a basal mechanism for tumor proliferation, migration and angiogenesis.

CD24 is a mucin-like adhesion molecule expressed in neutrophils, pre-B lymphocytes and a large number of solid tumors. Functionally, CD24 increases the metastatic potential of malignant cells due the fact that is identified as a P-selectin ligand, an adhesion receptor on activated endothelial cells and platelets. Lim and $\mathrm{Oh}$ investigated the role of CD24 in epitheliums of various human neoplasms and showed that intracytoplasmic CD24 expression was strongly associated with adenocarcinoma of the colon, stomach, gall bladder and ovaries. $\beta$-1 Integrin is a potential oral SC marker, expressed in basal keratinocytes and reduced regulation when the cells leave the basal layer. Human keratinocytes can be classified by the degree of their binding to type IV collagen, a natural ligand of $\beta-1$ integrin on two types. Fast-binding keratinocytes resemble SC while slow-binding keratinocytes with low levels of integrins $\beta-1$ behave as late ATC indicating that integrin $\beta-1$ for the maintenance of keratinocytes in the undifferentiated state is necessary. The main disadvantage of integrins $\beta-1$ as SC markers is the lack of specificity since about 20 $45 \%$ of basal keratinocytes exhibit high integrin $\beta-1$ expression [3].

Transcription Factors Oct3/4, Juice and Nanog Oct3/4, Juice play a key role in maintaining pluripotence and self-reproducibility of embryonic and adult SCs, by promoting self-renewal through interaction with Stat-3, Hesk-1 and Zic-3, and Signal molecules TCF-3, FGF-2 and LEFTV2. Oct3/4 is considered as one of the best indicators of the presence of stem cell properties. HNSCC cells that forming holoclones express a high level of these factors indicating that cancerous cells that have the expression of these factors have behaviors similar to SC [3].

E-cadherin binds to actin in the cytoskeleton by interacting with catechins. The non-regulation of E-cadherin, the suppressor of the invasion of molecules predicts a worsening of the prognosis. Decreased E-cadherin expression was observed in precancerous areas associated with the occurrence of multiple tumors. The loss of E-cadherin expression allows the release of $\beta$-catenin which acts as a transcription factor and the activation of Wnt times. E-Cadherin expression can be reduced by hypermethylation of the CDH1 promoter enhancing the metastatic potential of hypermethylated clones. E-cadherin-methylated tumor cells can be focused on invasive fronts spread to the lymph nodes and then become demethylated again regenerating the expression of E-cadherin. Therefore, methylation is reversible in OSCC progression which is very important because E-cadherin can favor the growth of metastatic tumor cells and activate pathways to avoid apoptosis [3].

\section{Stem Cells of the Head and Neck Cancer}

Cancer stem cells (CSCs) are tumor cells that have stem cell features such as self-reproducibility, high migration capacity, drug resistance and high proliferation capability. Studies on HNSCC cell lines are more prevalent than those on primary tumors which can be explained by the difficulties in cultivating fresh samples and high cost of tests. In fact, about ten major studies of HNSCC cell lines have been written in the last 6-7 years while only four articles describe experiments on primary head and neck tumors, one of which is a review article. Head and neck cancer is the sixth most common cancer and about 300,000 new cases are diagnosed every year. Despite progress in treatment, the 5-year survival rate for this cancer has not improved over the past 30 years. Progress in treatment and prognosis for HNSCC are limited by understanding tumor growth and progression. Human tumors including HNSCC represent heterogeneous histology and an additional concept is that the cancer contains subpopulations of cancer cells with high tumorigenic capacity so called cancer stem cells (CSCs). This heterogeneity is attributed to the clonal expansion process in 
which different clones are continuously produced and interpreted not as a result of genetic alteration but because of different ability to proliferate individual tumor clones. In his review Costea et al. [5] described the possible CSC origin that can support the hierarchy of the proliferative structure of the squamous cell carcinoma (OSCC). A more accepted hypothesis is that CSC can be stimulated by normal somatic stem cells. The normal oral epithelium has a regeneration rate of about 14-24 days, most epithelial cells do not exist for long enough to accumulate the genetic changes necessary for the development of the OSCC. In the hierarchical structure of stem cells present in the human oral epithelium it is clear that stem cells are the only long-lived inhabitants of the oral epithelium and, therefore, the only cells capable of accumulating the required number of genetic changes for the development of malignancy. It has been estimated that it takes three to six genetic events to transform a normal human cell into a cancer cell (Figure 3) [8].

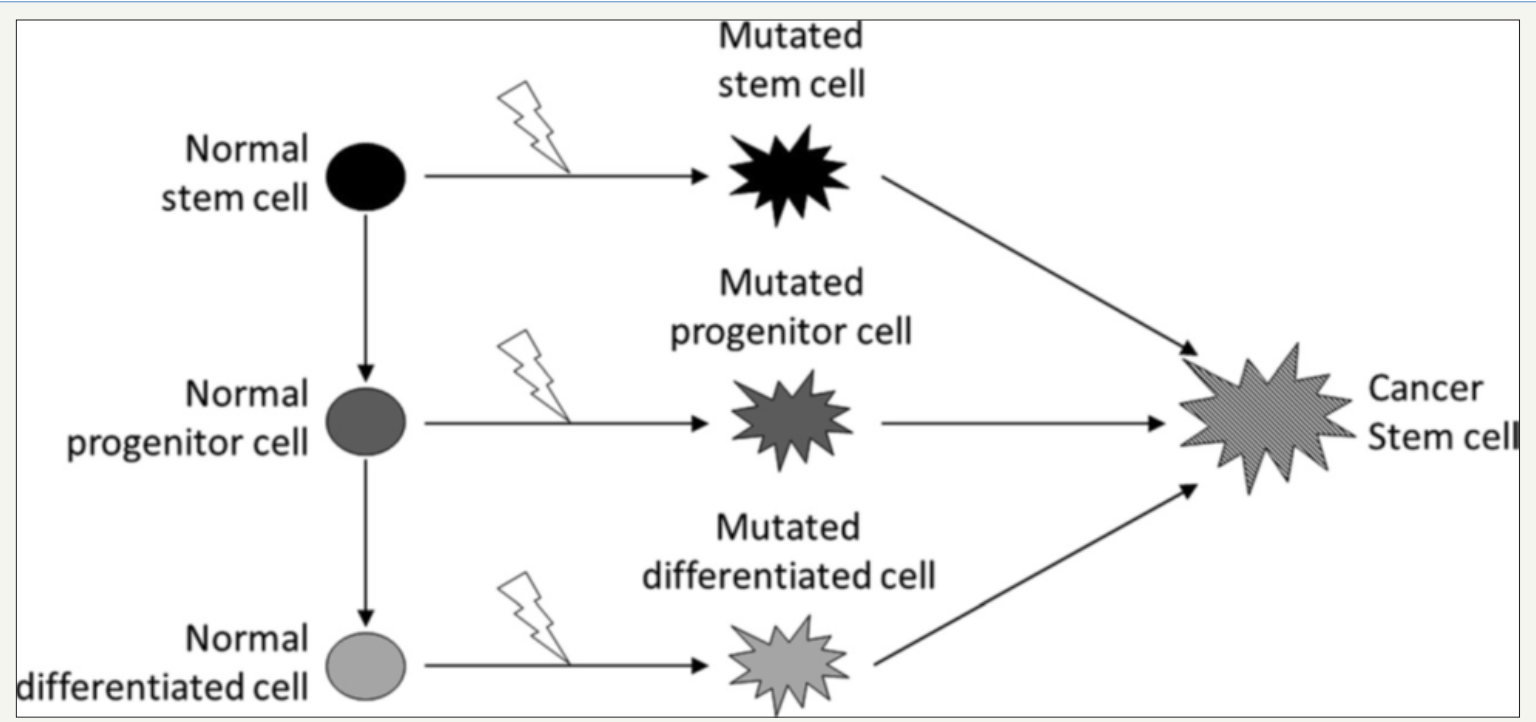

Figure 3: Potential origin for cancer stem cells includes normal stem cells, progenitor cells or completely differentiated cells. In order to produce the CSC, progenitor and fully differentiated cells must mutate to reactivate the genes responsible for increased proliferative activity, cell division and dedifferentiation.
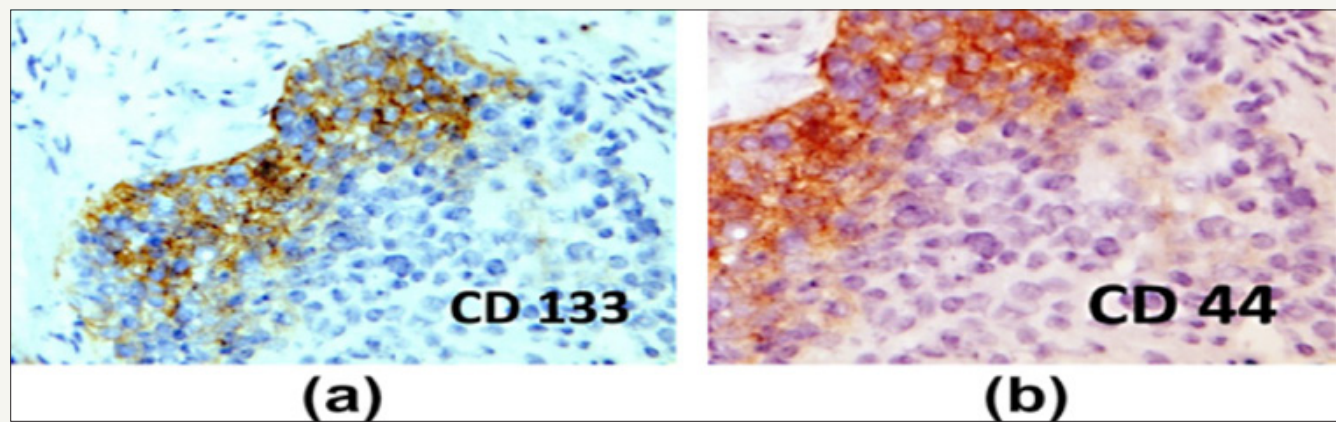

Figure 4: Immunohistochemistry for CD133 (a) and CD44 (b) markers of stem cells, isolated by tumor biopsy.

Calif. Et al. published data representing a direct link between existing genetic alteration and malignant phenotypic progression in OSCC and presented them as a model of genetic progression. This model is supported in vivo experimental animal studies of carcinogenesis of the skin indicating that exposure to the initial agents causes cellular changes that are retained within the tissue over an extended period of time. Another hypothesis supported by both in vitro and in vivo experiments suggests that oncogenic events may occur in keratinocytes derived from primitive stem cells and can cause renewal of stem cell capacity for self-regeneration and reduce terminal differentiation. More than $90 \%$ of all human neoplasms originate from the epithelium. In normal epithelial tissues the stem cells are usually found in the basal layer and they perform an asymmetric division that allows self-restoration of stem cells and differentiation in terminal cells. The degree of carcinoma differentiation depends on the ratio of undifferentiated tumor stem cells, the maturity stages of most cells in the main tumor mass and the ability of some cells to differentiate themselves. The hierarchical structure of stem cells present in the human epithelium indicates that the stem cells are a unique long-term cell and the only cells capable of accumulating the necessary number of genetic changes for malignant development. Obviously, tumor growth is associated with pattern and amplification patterns similar to those in normal tissues. There are six acquired carcinoma marks-unlimited replication of cells, self-sufficient growth, avoidance of apoptosis, insensitivity to signals against growth, sustained angiogenesis, invasion of surrounding tissues and metastases. Identification of carcinoma stem cells in head and neck tumors is the main goal of the stem cell biology agenda as well as the detection of key factors involved in self-renewal and differentiation patterns. However, it must be taken into 
account that there are still open controversies about the role of CSC in the development and progression of the tumor. In addition, biomolecular markers that define less subpopulations of the CSC show great variability that makes it difficult to characterize them and study tumor biology. Preliminary immunohistochemical results of some continuous studies (Figure 4) on the primary HNSCC sections provide additional evidence for a development hierarchy in HNSCC similar to that in normal squamous mucous membranes. Good to moderately differentiated HNSCC demonstrate a cellular organization with a basal layer differentiation with immature morphology cells towards the atypical layer with the appearance of more mature squamous morphology [9].

An analysis of the distribution of CD44 in well or moderately differentiated tumors suggests a clear redistribution topography in favor of strong positive layers of basal cells in contrast to several keratinized and differentiated cells in the higher layers in which this marker was absent (Figure 5). These findings provide additional evidence that HNSCC is organized into a development hierarchy as predicted by the CSC theory of carcinogenesis and indicating the possible value of CD44 in the HNSCC [9].

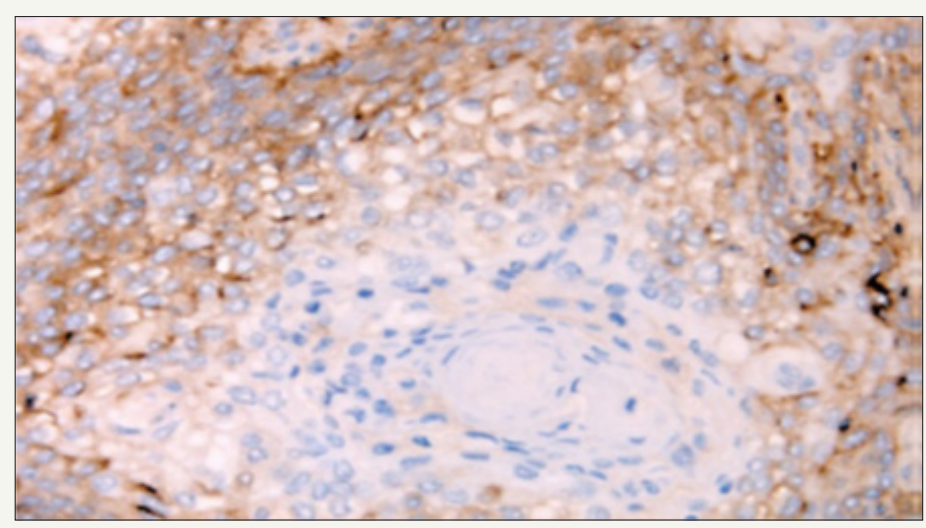

Figure 5: An analysis of the distribution of CD44 in moderately or highly differentiated tumors, indicating a clear topographic distribution of CD44-positive basal cell cells, while in a more differentiated and keratinized tissue this marker is absent.

Goodell et al. isolate a group of primitive stem cells with prolonged proliferation which differs from the main group of cells and is known as "sidepopulation" (SP). Zhang et al. demonstrated the presence of SP in most OSCC carcinoma analyzing OSC cell lines and primary tumors. They observed that SP were very variable among OSC samples from $0.1 \%$ to $10 \%$ of the cell population. Their proliferation rate has given a minimal contribution to tumor growth. Several SP cells were accumulated in the G1 phase while several non-SP cells were in the $S$ phase. SP cells showed a smaller squamous pattern of differentiation, lower expression CK13 and induction and greater expression of CK19 markers of stem cells. These results show that SP possesses the characteristics of stem cells and phenotypes due the fact that it can also regenerate the population of both SP and non-SP cells in vitro and in vivo. At the same time heterogeneous distribution of CD44 and Bmi- 1 suggests the existence of subpopulation in SP cells. Recognition of SP cells can help isolate the CSC, Wang et al. accented that while SP cells have many stem cell properties special surface markers are needed to differentiate CSC types in different tissues. They observed the presence of SP with stem properties studying cancer-cell lines purified from 5 nasopharyngeal carcinoma. From cultures in vitro, these cells could develop tumors in NOD/SCID mice. Tabor i sar. recently published a study that provides evidence of the theory of stem cell cancer showing that SP can produce tumors in the animal model while non-SP can't; they pointed out that SP represents cells with the features of stem cell carcinoma and provided a method by which CSC can be isolated and studied.

The known property of normal stem cells is their dependence on their micro-circuits or "niche", in order to maintain their undif- ferentiated state, while maintaining their proliferation and differentiation potential. Cell-cell interactions through direct contact or secretory factors support the survival and maintenance of stamina resistance in cancer and normal tissues. Several studies indicate an important role of stroma in the survival of epithelial stem cells and their controlled behavior; In fact, recent studies show that cancer is not just a disease of the transformed epithelium but also under the influence and dependence of the stromal environment. Malignant progression of oral epithelial cells appears to be associated with damage to the micro-environment associated with activation of fibroblasts or conversion to myofibroblast phenotypes. Activated myofibroblasts can produce proinvasive signals for transformed oral keratinocytes in the presence of diffused or solid matrix molecules by direct cell-cell contact or by a combination of both. De Costea et al. [5] have suggested in their review that the present and mesenchymal stem cells derived from the bone marrow are precursors of stroma associated with HNSCC and contribute to blood and lymphatic angiogenesis. Perivascular niches have been identified in neuronal tumor stem cells, however, until recently, it was not known whether head and neck cancer cells were localized in the immediate vicinity of blood vessels; Krishnamurhi et al. [10] recently demonstrated in vitro assays that factors excreted by endothelial cells promote self-restoration of the CSC head and neck and that about $80 \%$ of these cells were located next to the blood vessels in human tumors suggesting survival and self-regeneration of SC from endothelial cells and the existence of perivascular niches in the HNSCC.

Different markers and methodologies are explored to identify the CSC; however, no single marker or method can provide unam- 
biguous CSC identification in HNSCC. Thus, a combination of surface markers and proliferation rates make clear the definition of the CSC. It is known that the in vitro tumor sphere for the enrichment and isolation of CSC can't simulate the in vivo situation well, since the maintenance of a small CSC population in the cancer requires a specific micro-circulation; For now, the serial transplant method is generally considered a gold standard for the isolation of a small population of highly malignant cells from solid tumors. Chen et al. has found an increased expression of stem markers between the stem cell line and the CSC in the malignant stem cells in the original tumors. This may be due to the enrichment of all highly malignant CSCs from established cell lines in the test area while serial transplantation methods can only enrich the original CSCs that produce tumors in vivo. Interestingly, xenograft cells have also shown increased metastatic ability suggesting that enriched subpopulation may also show metastatic ability [9].

Numerous markers such as CD44, CD133, ALDH1, etc., and their numerous variants are also found in different proportions in the HNSCC. Thus, e.g. the presence of CD44v6 and v10 is largely associated with short survival time. Faber and colleagues underlined the role of CD44 as a diagnostic and prognostic factor in HNSCC and possible use as a potential therapeutic target [9].

\section{HNSCC sensitivity}

There are two major models that attempt to explain the progression of squamous cell tumors of the head and neck: stochastic clonal evolution model and CSC hierarchical model. According to the first, all tumor cells have the same tumor propagation ability and its consequent morphological heterogeneity is explained by aberrant cell differentiation due to genetic/epigenetic instability of tumor cells. The CSC model which applies the biological principles of stem cells suggests CSC maintenance of tumor proliferation by generating undifferentiated and more differentiated cells that form tumor mass. Accordingly, each tumor formation model suggests a different therapeutic approach. We can conclude that there is a clonal relationship between the genetic profile of the cancer and the surrounding area (Figure 6) [10].

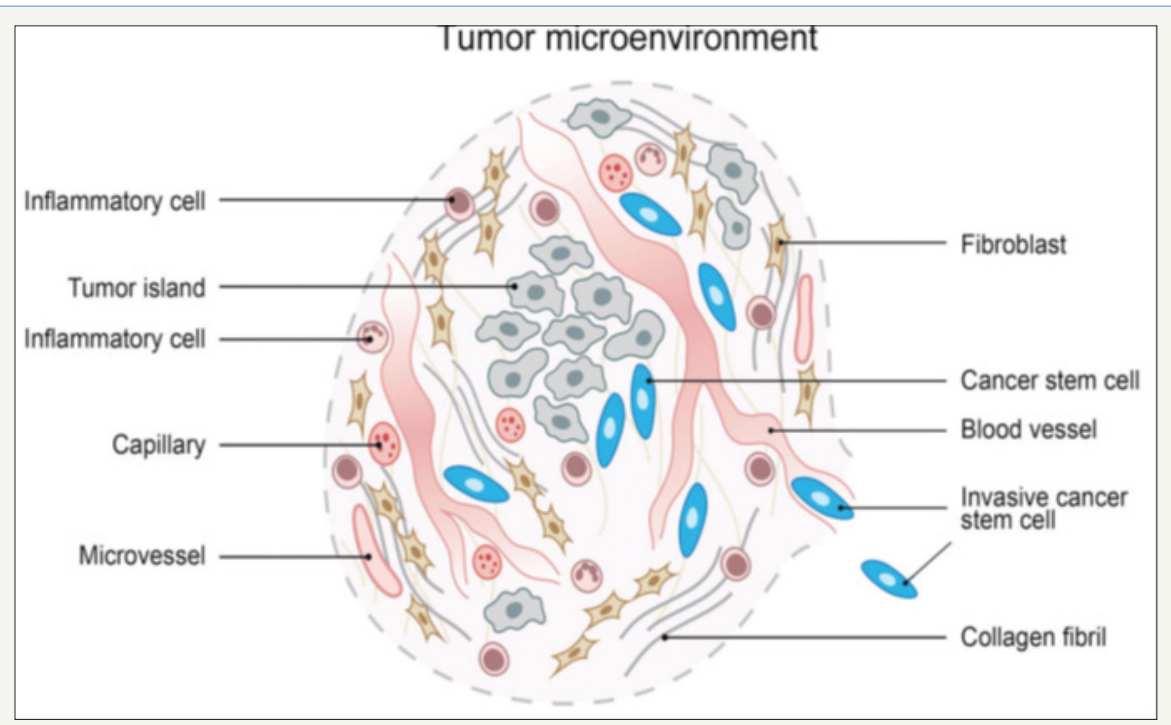

Figure 6: Cancer tissue is a complex "organ". The micro-environment of the tumor tissue is composed of various cells including tumor cells, cancer cells, inflammatory cells and cancer-associated fibroblasts together with blood vessels. Carcinoma stem cells are rare cells found primarily in the invasive edge of the tumor near the blood vessels, i.e. in a supportive perivascular niche.

In general, cancer occurs through genetic/epigenetic changes accumulated in a genome that appear in cancer cells and signal pathways along with genetic predisposition are also present. It is known that the squamous cell carcinoma of the head and neck appear in the pharynx, the oral cavity and throat and they are the sixth leading cancer around the world and their most important risk factors are smoking, alcohol consumption and, in particular, oropharynx tumors are caused by human papillomavirus (HPV). Tobacco use and alcohol consumption are well-established risk factors for these tumors. For smoking, the dose-response trend is known. Relative risk of development of laryngeal and oropharynx carcinoma is 1.8 and 1.3 , respectively for people who smoke $>30$ cigarettes per day compared to non-smokers. Alcohol consumption is also associated with increased risk of HNC (head and neck cancer). For people who consume $>4$ drinks $(=47.5 \mathrm{~g}$ of pure ethanol) daily the relative risks of developing laryngeal and oropharyngeal carcinoma are 4.5 and 7.2, respectively compared to non-drinking. A synergistic effect is observed in people who smoke tobacco and drink alcohol. The relative risks of developing oropharynx and laryngeal carcinoma are 34.6 and 21.2 respectively in those who smoke $>30$ cigarettes per day and consume $>4$ drinks per week.

HPV-positive and HPV-negative tumors represent various clinical, pathological and molecular entities; the first group of tumors has an increased incidence in the population under 60 , representing rare mutations in p53, which is very common in HPVnegative carcinoma. The HPV infection displays orofarinks as a preselection site and it is possible to find a connection with oral sexual habits, and HPV-positive tumors have a better prognosis than the HPV-negative group. Then, diet, poor oral health, exposure to carcinogenic effects of the environment and genetic polymorphism 
in carcinogenic metabolising enzymes (ALDH, GST) and DNA repair genes are potential risk factors for HNSCC, too. It is known that hypoxia is strongly associated with the aggressiveness of the cancer. Invasion is an important sign of cancer that involves interactions between the micro-environment and cancer cells. Hypoxia, a low level of oxygen is associated with increased invasion and metastases in many cancers.

The ARCAGE project, one of the largest multicentre genetic epidemiological studies on upper aerodigestion cancers (UADT) in Europe published in Cancer Research 2009 had its main goal of exploring the role of genetic variations in relation to the metabolism of alcohol and tobacco smoke carcinogens in the development of cancer UADT. This study was inspired by the idea that there are genetic polymorphisms involved in tobacco carcinogen and tobacco carbohydrate metabolism and DNA polymorphism (SNPs) research in these carcinomas can help to better understand the pathways leading to cancer. For example, Sabita et al. identified the association between years of smoking and HNSCC risk among CIP1A1 polymorphism cases; and stuff, heavy smokers (more than $>15$ years) have shown increased risk of HNSCC in relation to mutations $\mathrm{m} 1$ and $\mathrm{m} 2$. It is clear that biometabolism of the gene can play a significant role in the transformation of benign lesions into malignancies [10].

\section{Clinical and therapeutic implications of CSC in head and neck cancer}

Therapeutic modalities such as surgery, radiation, chemotherapy and combinations of each of them are used in the treatment of head and neck cancer but at the same time the scientific community is in the process of improving early detection and prevention of HNSCC. One of the keys to failing treatment can be explained by the presence of CSC that can avoid current therapeutic strategies. In fact, specific CSC markers such as ALDH1, CD44 and Bmi1 have demonstrated promising results in detection and a new therapeutic protocol.

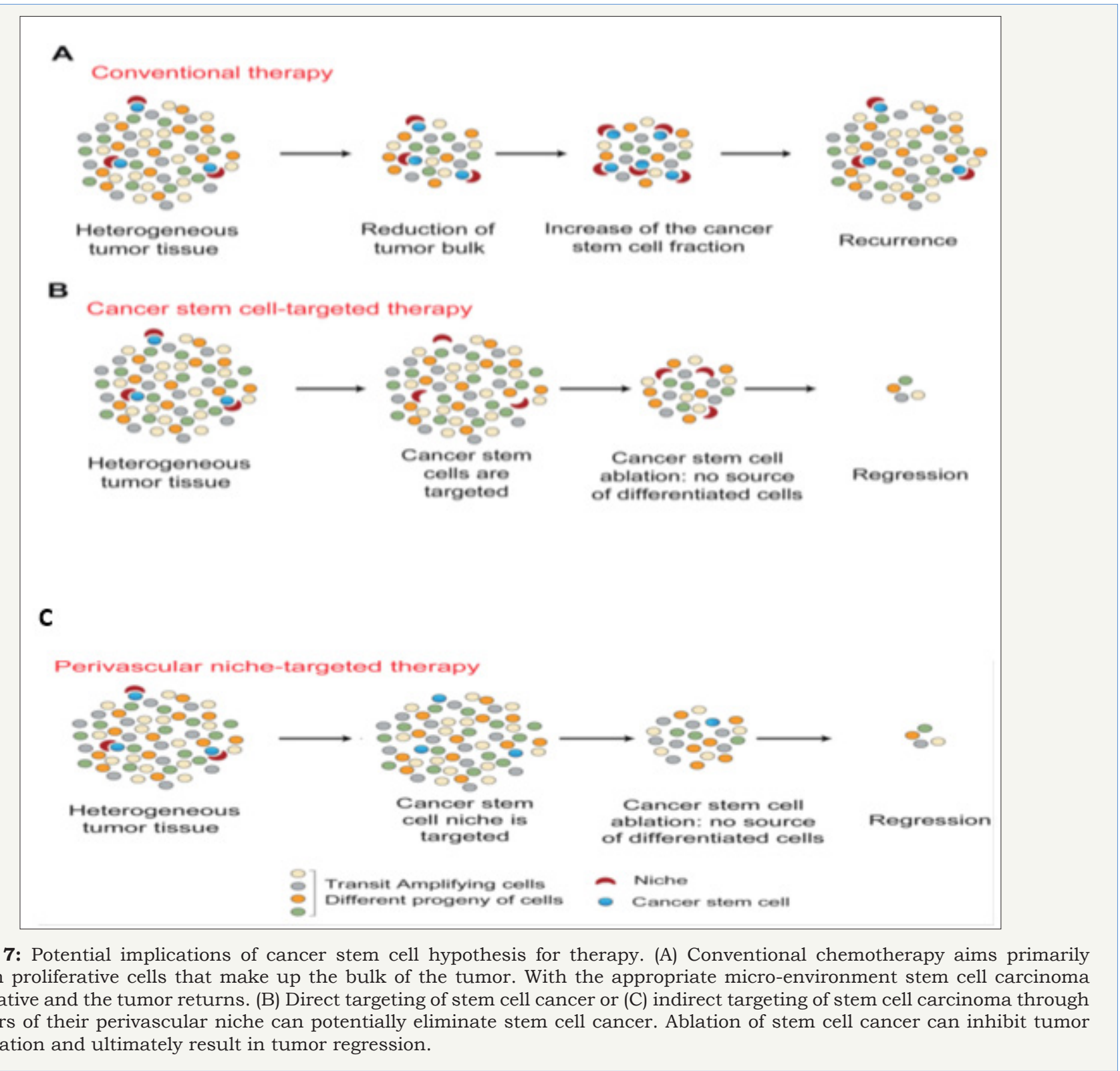


Strong epithelial tumors are the main cause of carcinoma death and have now been proven to be managed by a small subpopulation of malignant stem cells. In the normal epithelium the stem and amplification cells are divided in different ways, have different apoptotic sensitivity and different resistance to drugs; and, the persistence of such differences between malignant stem and amplification cells can affect therapeutic outcomes. Therefore, the identification of self-renewal and differential traits is needed for monitoring or better targeting, therapeutic intervention on the CSC. The existence of a hierarchical stem cell pattern suggests the possible achievement of a change in the asymmetric division which leads to the loss of tumor stem cells. It is suggested that normal and malignant stem cells have similar self-regeneration mechanisms, further, a focus on a mechanism that controls the asymmetric division of malignant stem cells can help develop new strategies for their therapeutic manipulation. The ability to identify stem cell cancer should lead to the development of more specific treatments for the treatment of tumors (Figure 7) [10].

In fact, by comparing the gene expression of the CSC, the tumor cell population which make the main tumor mass, normal stem cells and normal tissue it is possible to identify therapeutic methods that primarily attack the CSC. The first successful targeted therapy (EGFR-specific antibodies) shows that the understanding of molecular pathways found in the HNSCC base is improved and give valuable new protocol treatment.

Mitochondria appeared as an intriguing target for anti-cancer drugs inherent in the vast majority, if not all types of tumors. Drugs targeting mitochondria and enhancing anti-cancer activities have become the focus of recent research because of their great clinical potential (which has not been used up to now). The exceptional potential of mitochondria as a target of anti-carcinogenic agents is corroborated by the discouraging conclusion that even tumors of the same type in individual patients differ in numerous mutations. This is in line with the idea of personalized therapy, in line with the thought that tumors are unlikely to be treated with agents targeting only one gene or one route. This leads to mitochondria, potential target mass in all tumors. This led to the definition of a new class of anti-cancer drugs that act with mitochondrial "destabilization" and are called "mythocins". Mittocans are classified into several classes based on their molecular mode of action. Classification begins with those that act on targets on the surface of mitochondria and end with those in the mitochondrial matrix [11].

\section{Imunotherapeutic approaches to targeting CSC head and neck cancer}

In addition to resistance to hemio and radio therapy treatments targeting the CSC in HNSCC must overcome another major obstacle: the mechanisms for avoiding the immune response of the organism present in the CSC. Actually, current immunotherapy is mainly based on the present antigens that induce $\mathrm{T}$ cells through dendritic cells. Or, generally, these antigens are selected and derived from tumor mass cells; they are not derived from CSCs that may not cause an immunogenic reaction. CSCs may also be potent in antigen potential due to reduced surface expression of human leukocyte antigen (HLA). Therefore, in a heterogeneous tumor entity, CSC can lead to failure of treatment and disease progression avoiding the onset of current immunotherapy. Considering HNSCC, a better understanding of the communication between the CSC and the immune system is crucial in order to develop specific targeted therapies. Recently, ALDH1 has been identified as a potential target. Among the CSC markers studied ALDH1 is the most specific CSC marker used to identify high tumor cells present in the HNSCC. ALDH1 is recognized as the source of the antigen that induces a humoral immune response in HNSCC (Figure 8). Also, the data presented by Liao et al showed that the immune system of the host can recognize and distinguish CSC with ALDH1 phenotype of non-CSC cells. In addition to ALDH1, other cancer antigens have been identified preferred by CSC: Ciclin A1 has been reported in the leukemia stem cells of acute myeloid leukemia while DNAJB8 has been identified as a new antigen in renal CSC. This specific expression of cancer antigen can allow us to target therapy specific to the CSC. Moreover, the development of ALDH1A1 peptide-based vaccine for therapy is a new area for future research in the HNSCC [12].

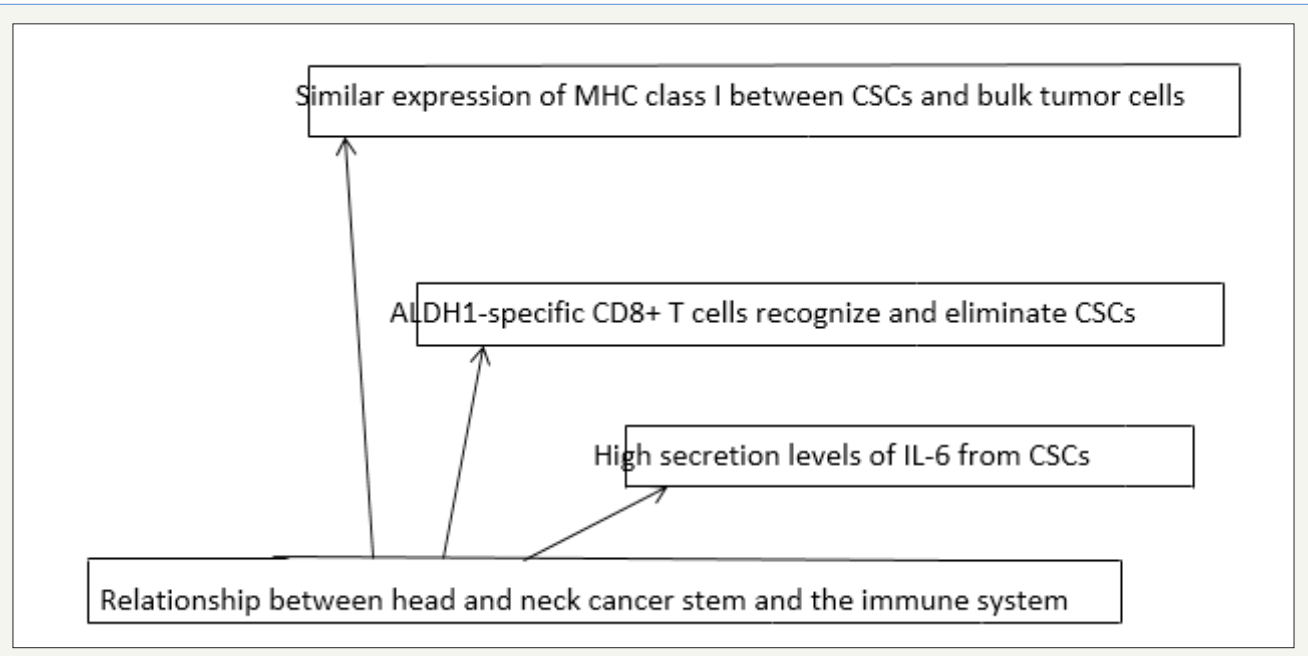

Figure 8: Immunotherapeutic approaches. CSCs: cancer stem cells; ALDH: Aldehyde dehydrogenase. 


\section{Conclusion}

Head and neck cancer is the sixth most common cancer and about 300,000 new cases are diagnosed every year making it the sixth most common malignant tumor in humans. Despite progress in treatment the 5-year survival rate for this cancer has not improved over the past 30 years. Progress in treatment and prognosis for HNSCC are limited by understanding tumor growth and progression. Since their discovery in 2007, CSCs in HNSCC have increased interest in further research into their properties: tumorigenicity, differentiation, proliferation and self-renewal. CSCs are substantially more resistant to traditional treatments such as radiation and chemotherapy contributing to potential metastases and recurrence of HNSCC. At the end, a better understanding of CSC biomarkers, signaling pathways and resistance mechanisms will improve therapies and outcomes through targeted interventions. Recently, the role of CSC in tumor neo-angiogenesis has been documented but we are still far from understanding molecular mechanisms that lead to carcinogenesis. However, there is a hope that the development of an in vitro and in vivo CSC model could provide a means for designing more effective cancer prevention, better diagnostic, prognostic and therapeutic strategies, especially for squamous cell head and neck cancer (HNSCC).

\section{References}

1. Reya T, Morrison SJ, Clarke MF, Weissman IL (2001) Stem cells, cancer and cancer stem cells. Nature 414: 105-111.

2. Clarke MF, Dick JE, Dirks PB, Eaves CJ, Jamieson CH, et al. (2006) Cancer stem cells-perspectives on current status and future directions: AACR workshop on cancer stem cells. Cancer Res 66(19): 9339-9344.

3. Zhaocheng Z, Manoel SF, Jacques EN (2012) The biology of head and neck cancer stem cells. Oral Oncology 48(1): 1-9.

4. Robert CZ, YaShu Z, Yufang S (2008) New hope for cancer treatment: Exploring the distinction between normal adult stem cells and cancer stem cells. Pharmacology \& Therapeutics 119(1): 74-82.

5. Costea DE, Tsinkalovsky O, Vintermyr OK, Johannessen AC, Mackenzie IC (2006) Cancer stem cells-new and potentially important targets for the therapy of oral squamous cell carcinoma. Oral Dis 12(5): 443-454.

6. Boman BM, Wicha MS (2008) Cancer stem cells: a step toward the cure. J Clin Oncol 26(17): 2795-2799.

7. Miguel ÁGM (2017) Cancer stem cells-Biopathology with reference to head and neck cancers (Chapter 2). Springer Science+Business Media BV, Germany.

8. Kaveh K, Nathan L, Reigh YL (2015) The role of cancer stem cells in head and neck squamous cell carcinoma and its clinical implications (Chapter 4). New Aspects in Molecular and Cellular Mechanisms of Human Carcinogenesis, USA.

9. Giuditta M, Oreste G (2012) Cancer stem cells hypothesis and stem cells in head and neck cancers. Cancer Treatment Reviews 38(5): 515-539.

10. Krishnamurthy S, Nör JE (2012) Head and neck cancer stem cells. J Dent Res 91(4): 334-340.

11. Jiri N, LanFeng D, Jakub R, Jaroslav T, Stephen JR (2012) Classification of mitocans, anti-cancer drugs acting on mitochondria. Mitochondrion 13(3): 199-208.

12. Benoîte M, Jean BG, Sophie E, Anne SW, Stéphanie S, et al. (2016) Targeting head and neck tumoral stem cells: From biological aspects to therapeutic perspectives. World J Stem Cells 8(1): 13-21.
Creative Commons Attribution 4.0

International License

For possible submissions Click Here

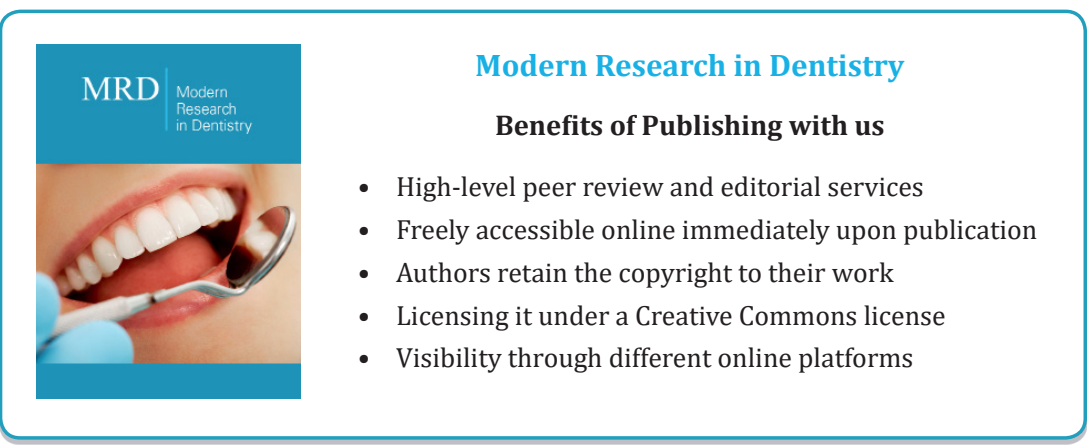

